\title{
Traumatic occlusion of the external iliac artery in a racing cyclist: a cause of ill defined leg pain
}

\author{
Alan J Taylor, William G Tennant, Mark E Batt, W Angus Wallace
}

\begin{abstract}
Stenosis of the external iliac artery in healthy athletes, although uncommon, has been reported in competition cyclists. A case of a racing cyclist whose chronic vague leg symptoms were incorrectly attributed to $L 4 / 5$ nerve root irritation is reported. This highlights the importance of clinical vascular testing when assessing ill defined leg pain. The role of trauma as a causative factor in this condition has not been previously documented.

(Br F Sports Med 1997;31:155-156)
\end{abstract}

Keywords: trauma; external iliac artery; endarterectomy; cycling

Stenosis of the external iliac artery (EIA) affecting competition cyclists was first reported by Walder et $a l^{1}$ and Mosimann et al. ${ }^{2}$ Since then less than 100 cases have appeared in the published literature. Increasing awareness of the condition on the continent has led to a recent proliferation of successful surgical procedures on professional cyclists (A Fotheringham, personal communication, 1996). Careful screening of athletes presenting with ill defined leg pain is required to identify the condition and to prevent delays in diagnosis or misdiagnosis.

\section{Case report}

A previously healthy 32 year old premier category racing cyclist presented complaining of a five year history of vague left leg pain, affecting the buttock, anterolateral thigh, calf and foot, together with a numb feeling in the middle three toes. Pain was worsened initially by cycling, swimming, walking, and more recently standing, sitting, and driving. The symptoms had been getting progressively worse since their onset. At the time of assessment the patient was unable to continue his work as an engineering fitter because of the pain experienced whilst standing, and had been unable to take part in competitive cycle racing for a number of months.

The patient was able to relate two specific Department of
Orthopaedics, Queens Medical Centre,

Nottingham NG7 2UH United Kingdom

A J Taylor

W G Tennant

M E Batt

W A Wallace

Correspondence to: Mr Taylor.

Accepted for publication 18 December 1996
Since that time, the patient had sought several specialist opinions, all of which had resulted in an unsubstantiated diagnosis of lumbar nerve root entrapment. Previous com- puterised tomography and magnetic resonance imaging of the lumbosacral spine had demonstrated no significant abnormality.

Physical examination demonstrated full and unrestricted painless lumbar movement with no palpable tenderness proximally or distally. Muscle strength and lengths were normal, with no sign of muscle imbalance. Straight leg raise and slump test were normal with no sensorimotor deficit. Examination of the lower limb pulses revealed the left femoral pulse to be barely palpable, with absent distal pulses. A clinical diagnosis of vascular insufficiency with claudication pain was made.

Resting ankle brachial pressure index (ABPI) on the left side was 0.56 and 1.12 on the right (normal $=1.1$ ). On treadmill testing, the patient developed claudication pain in the left calf at 40 metres, with a small reduction in post exercise ABPI. Lower limb arteriography demonstrated a short complete occlusion of the left EIA.

The patient underwent surgical vascular reconstruction. Operation was performed through a left retroperitoneal approach. The EIA was found to be of normal length, wall thickness, and diameter but was non pulsatile. There was a complete occlusion composed of both white and red thrombus densely adherent to the intima, typical of local trauma. There was no evidence of chronic atheromatous occlusive disease. Endarterectomy and vein patching were performed with immediate return of all distal pulses. The patient made a rapid recovery, was discharged five days after the operation and advised to refrain from training for three months.

\section{Discussion}

Arterial disease affecting racing cyclists has remained largely unreported in this country. Early in its course the patient usually complains of intermittent claudication of one limb, at a time of maximal strain-that is, climbing, sprinting, or time trialling, usually during competition or intensive training. Systolic ankle pressure may be normal at rest but markedly reduced after maximum exertion. Arteriography may show moderate stenosis $(<40 \%)$ and sinuous lengthening. ${ }^{34}$ Pathological findings have included stenotic intimal thickening due to connective tissue. ${ }^{4}$

Certain predisposing anatomical conditions such as an arterial branch to psoas, the cramped position on the bicycle, high training mileage, and abnormal haemodynamics during exertion may lead to this condition. ${ }^{34}$ Mosimann et al ${ }^{2}$ suggest that high cardiac output, arterial hypertension at maximal strain, 
and local turbulence in the EIA, combined with strong thigh flexion and an abnormally sinuous section of artery, may be the cause of a slow progressive stenotic intimal thickening. The high incidence of falls or crashes at competition level cycling has not previously been considered as a predisposing factor.

This case was atypical in that complete rather than partial occlusion had occurred, so that the patient had begun to feel symptoms during normal activity and not just maximal exercise.

It appears from the pathological findings that, despite the transient nature of the early symptoms, trauma may have been a factor in the development of the symptoms. As far as we are aware this is the first report of ischaemia in an athlete caused by trauma connected with cycling.

Despite its rarity, awareness of this condition is essential to prevent delays in diagnosis. Our case illustrates the importance of comprehensive evaluation of athletes. Vascular pathology must be considered when assessing patients complaining of vague unilateral leg pains during or after competition. Cyclists in particular may well be predisposed to stenosis or occlusion of the EIA.

1 Walder J, Mosimann F, Van Melle G, Mosimann R. A propos de l'endofibrose iliaque chez deux courers cyclists. Helv Chir Acta 1984;51:793-5.

2 Mosimann R, Walder J, Van Melle G. Stenotic intimal thickening of the external iliac artery. Illness of competition cyclists. Vascular Surgery 1985;19:258-63.

3 Chevalier JM, Enon B, Walder J, Barral X, Pillet J, Megret $A$, et al. L'endofibrose iliaque externe du cyclist du competition. Une pathologie arterielle meconnue. Annales de Chirurgie Vasculaire 1986;1:297-303.

4 Rousselet MC, Saint-Andre JP, L'Hoste P, Enon B, Megret A, Chevalier JM. Stenotic intimal thickening of the externa iliac artery in competition cyclists. Hum Pathol 1990;21 524-9.
Department of University Hospital of Wales, Heath Park, Cardiff CF4 4XW, United Kingdom I A Wright

N D Pugh

\section{Department of} Cardiology, University Hospital of Wales, Heath Park, Cardiff CF4 4XW, United Kingdom J Goodfellow A G Fraser

Department of Radiology, University Hospital of Wales, Heath Park, Cardiff CF4 4XW, United Kingdom

A M Wood

Department of Surgery, University Hospital of Wales, Heath Park, Cardifi CF4 4XW, United Kingdom

I F Lane

Correspondence to: Miss I A Wright, Department of Medical Physics and Bioengineering, University Hospital of Wales, Heath Park, Cardiff CF4 4XW, United Kingdom.

Accepted for publication 18 December 1996

\title{
Dynamic obstruction of the external iliac artery in endurance athletes and its relationship to endothelial function: the case of a long distance
}

\section{runner}

\author{
Isabel A Wright, Neil D Pugh, Jonathan Goodfellow, Andrew M Wood, Ian F Lane, \\ Alan G Fraser
}

\begin{abstract}
There have been recent reports of exercise induced claudication in endurance trained athletes attributed to narrowing of the external iliac artery. Most patients have been competitive cyclists, and intimal hyperplasia has been cited as the cause. The case is reported here of a long distance runner who presented with similar symptoms.
\end{abstract}

(Br F Sports Med 1997;31:156-158)

Keywords: external iliac artery; long distance runner; diabetes mellitus

Since the early 1980 s there have been several reports documenting stenoses of the external iliac artery (EIA) in competitive cyclists. ${ }^{1-3}$ They describe how intimal hyperplasia/ endofibrosis produces a narrowing of the EIA, leading to lower limb claudication at maximal effort. It is thought that intimal hyperplasia may be produced in response to repetitive arterial injury. Endarterectomy and vein patch angioplasty have been used to treat some cases. $^{2}$
Here we describe a long distance runner who presented with similar symptoms, apparently caused by arterial spasm.

\section{Case report}

A 39 year old female marathon runner presented with an 18 month history of acute pain in her right thigh and calf after running approximately 200 yards. She was a normotensive insulin dependent diabetic (of 18 months) and an ex-smoker ( 20 per day) from 12 years previously. Clinical examination was normal and there was no family history of cardiovascular disease.

Magnetic resonance imaging showed no evidence of lumbar canal stenosis or popliteal entrapment.

Resting Doppler studies showed a normal ankle/brachial systolic pressure index (ABPI) of 1.00 on the right and 1.06 on the left, with triphasic ankle waveforms bilaterally. There were raised velocities in the right common femoral artery (CFA) with a diffuse narrowing of the EIA as compared with the left on Duplex scan; however, this was not haemodynamically significant. Otherwise the lower limb vasculature appeared entirely normal. 